\title{
Role of nitrergic input in mechanically and chemically induced gastric relaxation in conscious dogs
}

\author{
Masafumi KIKKAWA ${ }^{1}$ \\ ${ }^{1}$ Department of Surgery, Division of Frontier Medical Science, Programs for Biomedical \\ Research, Graduate School of Biomedical Sciences, Hiroshima University, 1-2-3 \\ Kasumi, Minami-ku, Hiroshima 734-8551, Japan
}

\begin{abstract}
Our aim was to study a role of nitrergic input in gastric relaxation in conscious dogs. Proximal gastric motor responses to mechanical distension and chemical stimulation (a lipid meal orally) were evaluated by electronic barostat. Effect of $\mathrm{N}^{\mathrm{G}}$-monomethyl-Larginine acetate (L-NMMA, $5 \mathrm{mg} / \mathrm{kg}$ ) on these responses was studied. When mechanical stimulation was applied, we observed steep linear increases in intragastric pressure up to about $6 \mathrm{mmHg}$, then continued to increase gradually, and could be increased still further upon the addition of L-NMMA. Oral application of a lipid led to a prompt fall in intragastric pressure (gastric receptive relaxation; GRR). Lipid treatment also led to a considerable increase in gastric volume (means \pm S.D., $150.0 \pm 50.2 \mathrm{ml}$ ), this was followed by a plateau phase and a gradual return to baseline levels. Neither GRR nor the associated increase in gastric volume $(167.6 \pm 53.0 \mathrm{ml})$ was sensitive to treatment with L-NMMA. Our conclusion is that nitrergic input is necessary for mechanically induced gastric relaxation, but not for either GRR or chemically-induced gastric relaxation in conscious dogs.
\end{abstract}

Key words: barostat, gastric accommodation, gastric adaptive relaxation, gastric receptive relaxation, $\mathrm{N}^{\mathrm{G}}$-monomethyl-L-arginine acetate (L-NMMA)

\section{Introduction}

The stomach can be divided into two parts on purely functional grounds. This distinction relates to the proximal and distal stomach, and was first recognized by Cannon (Cannon, 1898). The distal portion of the stomach plays an important role in triturating food into small pieces, while the proximal stomach relaxes to receive ingested food with a minimal increase in intragastric pressure. This physiological response acts to increase reservoir capacity, and appears to involve two reflexes. Initially, when food passes through the pharynx and esophagus, the proximal stomach relaxes in what has been described as receptive relaxation (Cannon $e t$ al.,

Correspond to: Masazumi Okajima, M.D., Ph.D., Department of Surgery, Division of Frontier Medical Science, Programs for Biomedical Research, Graduate School of Biomedical Sciences, Hiroshima University, 1-2-3 Kasumi, Minami-ku, Hiroshima 734-8551, Japan

Phone: +81-82-257-5222 Fax: +81-82-257-5224 e-mail: mokajima@hiroshima-u.ac.jp 
1911). Then when food actually enters the stomach, a further relaxation phase occurs. This second phase is usually described as adaptive relaxation (or occasionally as gastric accommodation), and seems to represent a reflex relaxation of the gastric wall in response to either mechanical or chemical stimulation.

Gastrointestinal motility is believed to be regulated in the main by cholinergic nerves (Azpiroz et al., 1987; Desai et al., 1991) and to a lesser extent by non-adrenergic and noncholinergic (NANC) inhibitory nerves (Abrahamsson et al., 1973; Martinson et al., 1965). Recent evidence suggests that nitric oxide (NO) can serve as a key NANC inhibitory transmitter in the gastrointestinal tract (Desai et al., 1991; Mulemans et al., 1995; Uno et al., 1995; Paterson et al., 2000). Other studies indicate that mechanically induced gastric adaptive relaxation and gastric receptive relaxation in both the isolated guinea-pig stomach (Desai et al., 1991; Uno et $a l ., 1995)$ and the anesthetized dogs (Mulemans et al., 1995) are mediated by NO via vagal NANC inhibitory nerves. However, it is not yet known whether activation of a nitrergic input contributes to gastric adaptive relaxation and/or gastric receptive relaxation in the conscious state. We therefore set out to study the effects of nitrergic input on mechanically induced gastric adaptive relaxation and gastric receptive relaxation in conscious dogs.

Chemically induced gastric relaxation has been studied previously (Azpiroz et al., 1985; Azpiroz et al., 1986; Mulemans et al., 1995; Ropert1 et al., 1993; Undeland et al., 1995; McLaughlin et al., 1998). The post-prandial increase in the volume of the proximal stomach is about $350 \mathrm{ml}$, and in humans is largely dependent on the fat content of meals (Ropert1 et al., 1993; Undeland et al., 1995; McLaughlin et al., 1998). Unfortunately, there are few reports in which possible mechanisms of chemically induced gastric relaxation are described. In particular, little if anything is known about the suggestion that nitrergic input could have a key role in chemically induced gastric relaxation.

To our knowledge, this is the first study of the role of nitrergic input in both mechanically and chemically induced gastric adaptive relaxation, and in gastric receptive relaxation, to be conducted in conscious animals.

\section{Methods}

All procedures used in this study were approved by the Animal Ethics Committee of Hiroshima University, Japan.

We used an electronic barostat that accurately and continuously measures pressure and volume in the gastrointestinal tract (Azpiroz et al., 1985). This device provides particularly good estimates of the changes in gastric tone that occurs after liquid meals (Ropert et al., 1993). The barostat consists of an air insufflation system and a pressure transducer. Intragastric bag pressure can be maintained at a constant level by an external electronic feedback mechanism. The barostat was used according to standard guidelines issued in 1997 (Whitehead et al., 1997).

Nine adult mongrel dogs of both sexes (body weight, 10-14 kg) were operated. A gastric cannula $(\phi 8 \mathrm{~mm})$ was inserted into the lower corpus of the stomach. The cannula was exteriorized at the left side of the abdomen. After a postoperative recovery period, the following experiments were done while the animals were fully conscious. After the dogs had fasted for 24 


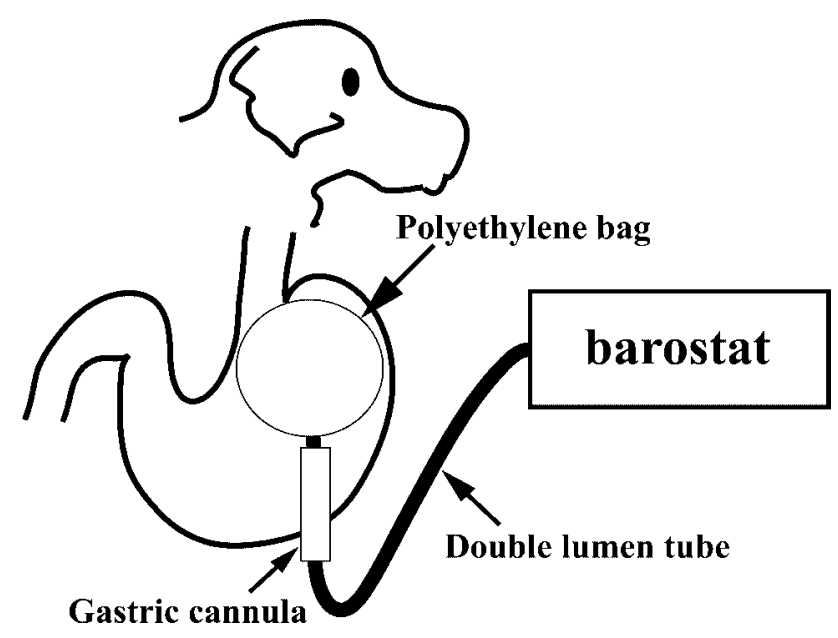

Fig. 1. Schematic representation of canine experimental model with barostat system. A polyethylene bag was positioned via a gastric cannula into the gastric fundus and was connected to a barostat by means of a double lumen tube. Gastric relaxation was measured by the barostat as increments in bag volume.

hours, an ultra thin polyethylene bag (size: $16 \times 14 \mathrm{~cm}$, maximum volume: $600 \mathrm{ml}$ ) was placed in the proximal stomach via the gastric cannula. The bag was connected to the barostat by means of a double-lumen polyvinyl tube (Fig. 1). After a stabilization period, the experiments described below were begun. We recorded any movements by the dogs throughout all of our experiments.

\section{Mechanical stimulation}

The intragastric polyethylene bag was insufflated at a constant rate of $90 \mathrm{ml} / \mathrm{min}$. The intragastric bag volumes and pressures were continuously recorded by the barostat.

To investigate possible physiological roles of the nitrergic pathway in mechanically induced gastric relaxation in conscious dogs, we administered $5 \mathrm{mg} / \mathrm{kg}$ (this was the maximum dose with no major cardiovascular effects) of $\mathrm{N}^{\mathrm{G}}$-monomethyl-L-arginine acetate (L-NMMA) as an inhibitor of nitrergic pathways (Tocris Cookson Inc., Ellisville, MO) to each animal intravenously. Ten minutes after the administration of L-NMMA, we insufflated the intragastric polyethylene bags at the constant rate of $90 \mathrm{ml} / \mathrm{min}$. Intragastric bag volumes and pressures were continuously recorded by the barostat.

\section{Chemical stimulation}

To study the effects of chemical stimulation, we examined the levels of proximal gastric relaxation induced by oral intakes of water or 20\% lipid (Intralipos, Yoshitomi Pharmaceutical Company, Japan) (composition per $100 \mathrm{ml}$ : $200 \mathrm{Kcal}, 20.0 \mathrm{~g}$ purified soybean oil, $2.2 \mathrm{~g}$ glycerin, and $1.2 \mathrm{~g}$ lecithin), or following intragastric administration of lipid via the gastric cannula to circumvent any possible pharyngeal and esophageal effects. The water and lipid sources were kept at room temperature to avoid thermal effects (Villanova et al., 1997). Intragastric bag 
pressures were maintained at $4 \mathrm{mmHg}$ throughout the experiment. After the intragastric bag volume had stabilized, water or lipid was given. We decided upon a per meal dose of $2 \mathrm{ml} / \mathrm{kg}$ to prevent bag expansion from being limited by larger volumes of liquid in the stomach. Intragastric bag volumes and pressures were continuously measured by the barostat until the intragastric bag volumes had returned to their baseline values.

To investigate possible physiological roles of the nitrergic pathway on chemically induced relaxation in conscious dogs, we administered L-NMMA (5 mg/ $\mathrm{kg})$ intravenously. Ten minutes after the administration of L-NMMA, we provided lipid $(2 \mathrm{ml} / \mathrm{kg})$ by the oral route. Intragastric bag pressures were maintained at $4 \mathrm{mmHg}$ throughout the experiment. Intragastric bag volumes and pressures were continuously measured by the barostat until the intragastric bag volumes had returned to their baseline values.

$\Delta \mathrm{V}$ max, which means the maximum volume increase of the stomach, was calculated by subtracting baseline volume from maximum volume.

\section{Statistical analysis}

Results are expressed as means \pm S.D. We used two-tailed Student's $t$-tests for paired observations to determine the statistical significance of any differences between mean values. $\mathrm{P}$ values of less than 0.05 were taken to be statistically significant.

\section{Results}

\section{Mechanical stimulation}

Insufflation of the intragastric bag resulted in a steep linear increase in the pressure/volume gradient. The following this linear increase in pressure volume gradient was followed by a more gradual and slower increase in the pressure volume gradient. In most experiments, the gradual increase in the pressure/volume gradient began when the intragastric pressure had reached approximately $6 \mathrm{mmHg}$ (Figs. 2A and 3). Small fluctuations in pressure were believed to represent the effects of respiration.

After administration of L-NMMA, the intragastric bag pressures were similar to those of the control until pressures of approximately $6 \mathrm{mmHg}$ were attained. Beyond this point, intragastric bag pressures were significantly higher in L-NMMA treated dogs than in the corresponding controls (Figs. 2B and 3).

\section{Chemical stimulation}

We detected a prompt fall in intragastric pressure after water or lipid ingestion, indicative of receptive relaxation (Figs. $4 \mathrm{~A}$ and $5 \mathrm{~B}$ ). There was no sign of receptive relaxation when lipid was infused directly into the stomach, however (Fig. 5A). The ingestion of water caused minimal gastric relaxation $(\Delta \operatorname{Vmax}: 41.6 \pm 24.0 \mathrm{ml}$ ), and stomach volumes returned to baseline levels within 5 min (Fig. 5B). Both lipid ingestion and lipid infusion caused considerable gastric relaxation ( $\Delta$ Vmax: $150.0 \pm 50.2 \mathrm{ml}$ and $153.6 \pm 25.2 \mathrm{ml}$, respectively) followed by a plateau phase (Figs. 4A and $5 \mathrm{~A}$ ) after which gastric volumes gradually returned to baseline levels (Fig. $4 \mathrm{~B})$. There was a significant difference in $\Delta \mathrm{Vmax}$ between the water ingestion group and the 
A

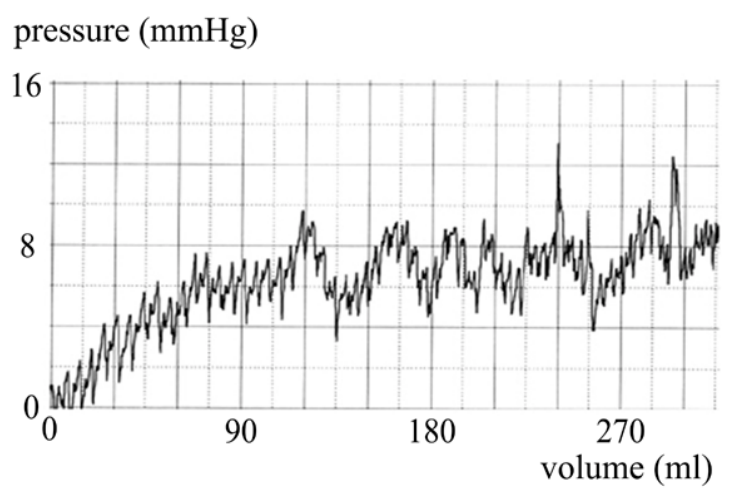

B

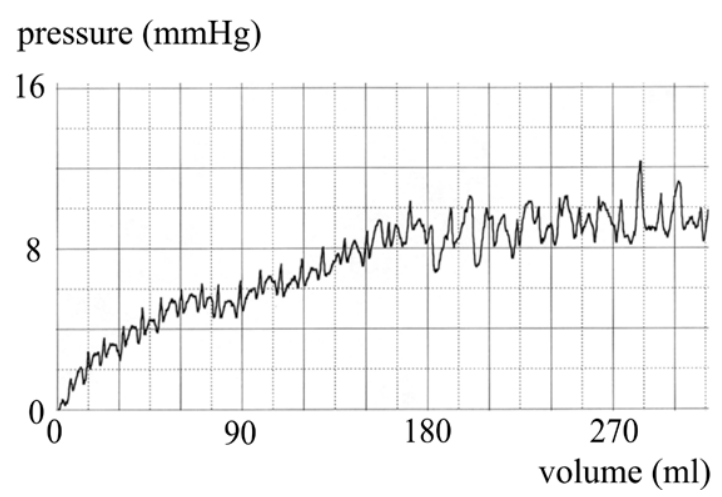

Fig. 2. Gastric relaxation response induced by mechanical stimulation. Representative examples of pressure-volume relationships are shown. (A) Control response and (B) after administration of L-NMMA. Insufflation of the intragastric bag resulted in a steep linear increase in the pressure/volume gradient and was followed by a gradual increase in the pressure/volume gradient, despite further gastric distension. Small fluctuations in pressure represent effects of respiration.

lipid ingestion group $(\mathrm{P}<0.005)$, but no apparent difference in $\Delta \operatorname{Vmax}$ between the lipid ingestion group and the lipid infusion group (Table 1). Small, regular fluctuations in pressure were assumed to represent the effects of respiration. Large, irregular increases in gastric pressure were found to result from movements of the dogs concerned (Figs. 4 and 5).

We detected no changes in fasting gastric tone consequent upon the i.v administration of 5 $\mathrm{mg} / \mathrm{kg}$ of L-NMMA. Lipid ingestion led to a prompt fall in intragastric pressure and to a rapid increase in gastric volume $(\Delta \operatorname{Vmax} ; 167.6 \pm 53.0 \mathrm{ml})$ followed by a plateau phase prior to a gradual return to baseline levels (Figs. 6 and 7). We were unable to see any significant differences in baseline gastric volumes between the lipid ingestion group and the L-NMMA + lipid ingestion group. L-NMMA had no discernible effect on the changes in gastric volume that we detected following lipid ingestion (Table 1). The post-ingestion time course of the gastric relaxation responses was similar in the two groups throughout our experiments (Fig. 7). 


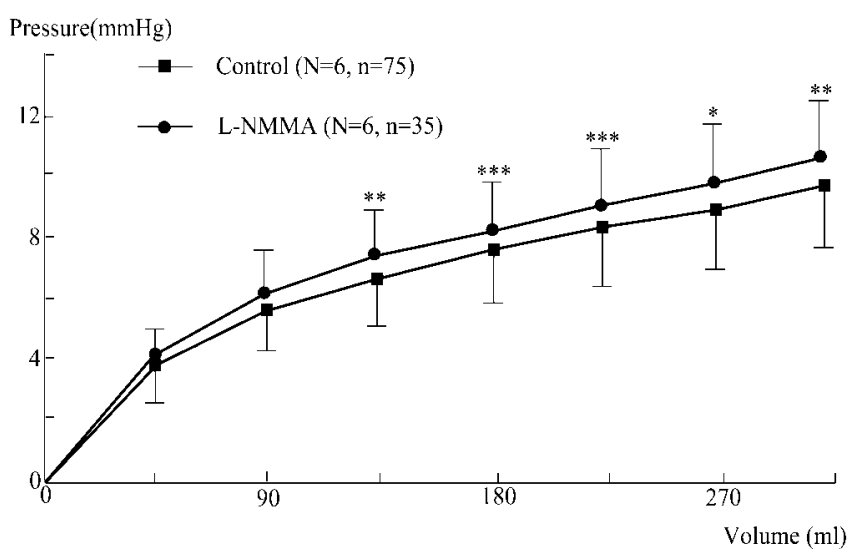

Fig. 3. Effect of L-NMMA on gastric relaxation induced by mechanical stimulation. Intragastric bag pressure was similar until the intragastric bag pressure reached about $6 \mathrm{mmHg}$. Thereafter, the intragastric bag pressure was significantly higher than control. $* \mathrm{P}<0.005$ vs Control. $* * \mathrm{P}<0.01$ vs Control. $* * * \mathrm{P}<0.05$ vs Control. Values represent means \pm S.D.

Table 1 Gastric relaxation response as measured by barostat

\begin{tabular}{lccc}
\hline & Baseline volume & Maximum volume & $\Delta \mathrm{V}$ Max \\
\hline Lipid ingestion $(\mathrm{N}=5, \mathrm{n}=26)$ & $49.9 \pm 15.4$ & $199.9 \pm 47.8$ & $150.0 \pm 50.2$ \\
Lipid infusion $(\mathrm{N}=5, \mathrm{n}=7)$ & $47.7 \pm 10.4$ & $201.3 \pm 22.4$ & $153.6 \pm 25.2$ \\
Water ingestion $(\mathrm{N}=5, \mathrm{n}=10)$ & $53.1 \pm 16.7$ & $94.7 \pm 23.3^{*}$ & $41.6 \pm 24.0^{*}$ \\
L-NMMA+Lipid ingestion $(\mathrm{N}=5, \mathrm{n}=15)$ & $47.7 \pm 18.4$ & $215.3 \pm 53.8$ & $167.6 \pm 53.0$ \\
\hline
\end{tabular}

All numbers are given as means \pm S.D. ${ }^{*} \mathrm{P}<0.005$ vs lipid ingestion group.

\section{Discussion}

The proximal stomach relaxes when food enters the stomach. This gastric relaxation, referred to as adaptive relaxation (or on occasions as gastric accommodation), appears to be a gastric wall reflex that responds to either mechanical or chemical stimulation.

In our control studies of mechanical stimulation, insufflation of air initially increased pressure in the proximal stomach in a linear fashion. This was followed by a gradual rise in intragastric pressure, despite continuing gastric insufflation. In most experiments, the pressure/volume gradient leveled off whenever the intragastric pressure had reached approximately $6 \mathrm{mmHg}$. This leveling off of intragastric pressure represents mechanically induced gastric adaptive relaxation. We chose $4 \mathrm{mmHg}$ as the maintenance pressure in our chemical stimulation studies because mechanically induced gastric adaptive relaxation did not occur at this value. Two recent studies have led their authors to suggest that adaptive relaxation is mediated by NO via vagal NANC inhibitory nerves in isolated guinea-pig stomach in the presence of atropine and guanethidine (Desai et al., 1991; Uno et al., 1995). It has not been determined whether the activation of nitrergic input leads to adaptive relaxation in vivo in conscious animals. We therefore examined the role of nitrergic input in gastric adaptive relaxation in conscious dogs. 
A

Volume (ml)

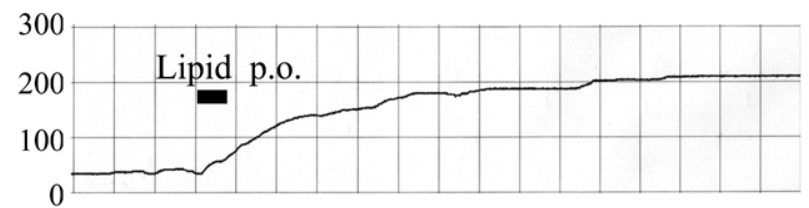

Pressure $(\mathrm{mmHg})$

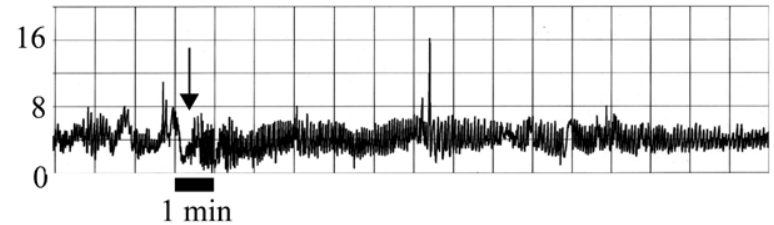

B

Volume (ml)

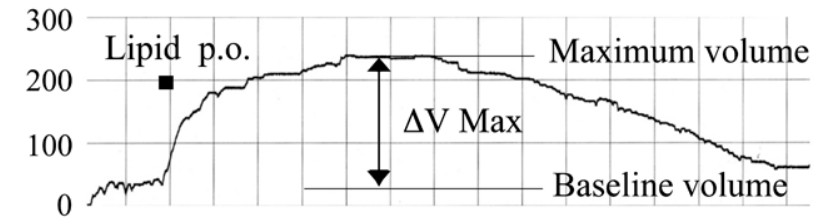

Pressure $(\mathrm{mmHg})$

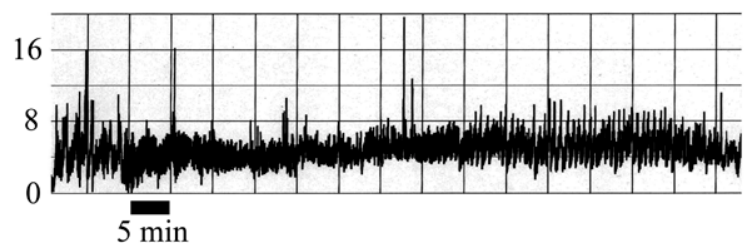

Fig. 4. Gastric relaxation response induced by lipid ingestion. Intragastric bag pressure was maintained at $4 \mathrm{mmHg}$. (A) There was a prompt fall in intragastric pressure after lipid ingestion, indicating receptive relaxation (The arrow represents gastric receptive relaxation). (B) Lipid ingestion induced considerable gastric relaxation followed by a plateau phase, and gastric volume gradually returned to the baseline level. Small and regular fluctuations in pressure represent effects of respiration. Large, irregular increases in gastric pressure were found to result from movements of the dogs concerned.

After treatment with L-NMMA, intragastric pressures were significantly higher than the controls after an intragastric pressure in excess of $6 \mathrm{mmHg}$ had been achieved, thus indicating that nitrergic pathway may well regulate adaptive relaxation. Our results are consistent with those of previous studies (Desai et al., 1991; Uno et al., 1995).

Patients with functional dyspepsia are impaired with respect to adaptive relaxation (Coffin $e t$ al., 1994; Kim et al., 2001), as well as suffering from abnormalities in their intragastric distribution of food that are independent of their gastric emptying rates (Troncon et al., 1994). Glyceryl trinitrate tablets, which generate NO, alleviate these abdominal symptoms in functional dyspepsia patients (Hausken et al., 1994); this may indicate that NO is capable of acting as a key 
A

volume (ml)

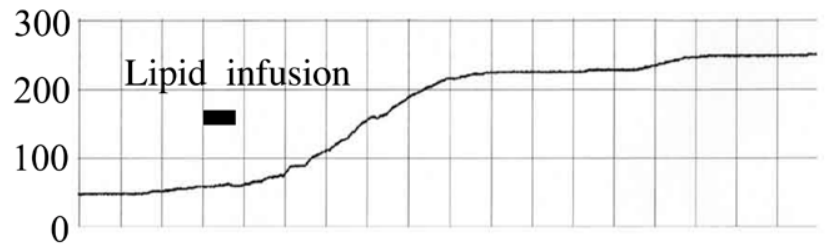

pressure $(\mathrm{mmHg})$

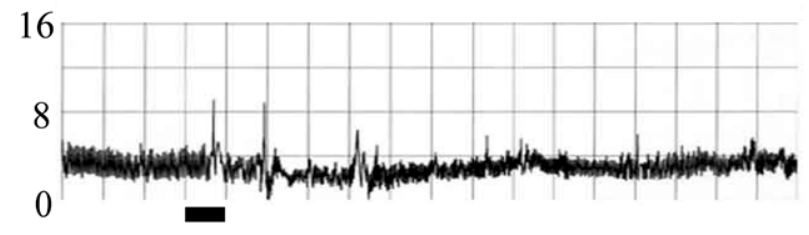

$1 \mathrm{~min}$

B

volume (ml)

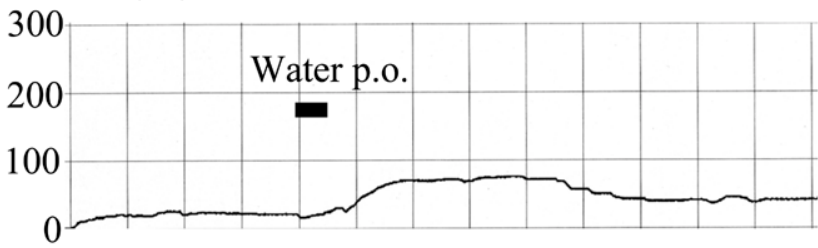

pressure $(\mathrm{mmHg})$

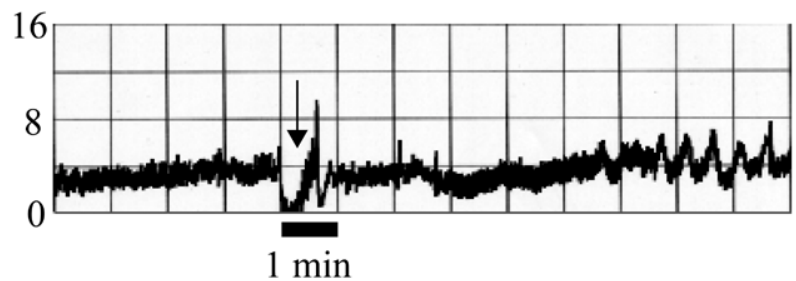

Fig. 5. Gastric relaxation responses induced by lipid infusion or water ingestion. (A) Lipid infusion into the stomach induced substantial gastric relaxation, but a prompt fall in intragastric pressure, indicating receptive relaxation, did not occur. (B) Water ingestion induced minimal gastric relaxation, and gastric volume returned to the baseline level within $5 \mathrm{~min}$. The arrow represents gastric receptive relaxation.

transmitter in the processes leading to adaptive relaxation, and that it is effective in alleviating functional dyspepsia simply because it regulates this process. Overall our results suggest that nitrergic input is necessary for mechanically induced gastric relaxation in conscious dogs.

Our results also show that both the ingestion and infusion of lipid can lead to substantial and prolonged gastric relaxation, whereas water ingestion led to a relatively brief and much 
volume (ml)

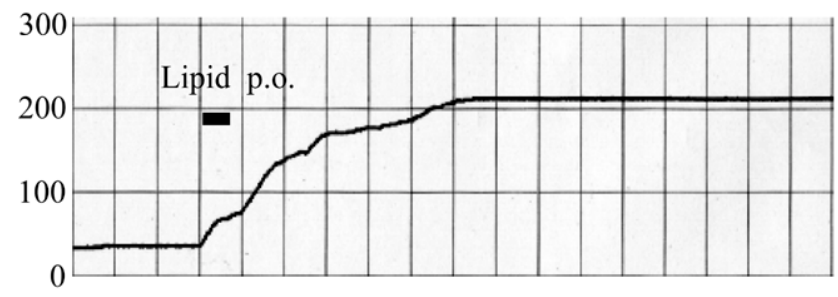

pressure $(\mathrm{mmHg})$

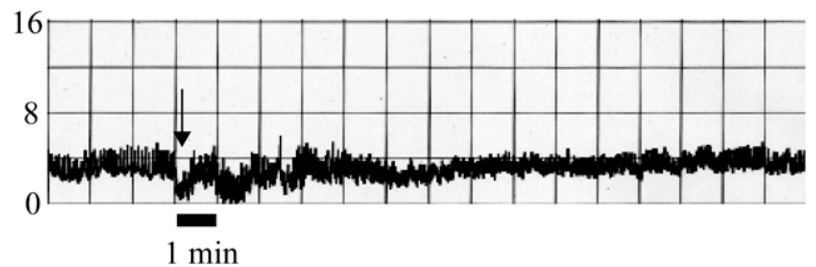

Fig. 6. Gastric relaxation response induced by lipid ingestion after administration of LNMMA. There was a prompt fall in intragastric pressure after lipid ingestion, indicating receptive relaxation, and gastric volume rapidly increased followed by a plateau phase. The arrow indicates gastric receptive relaxation.

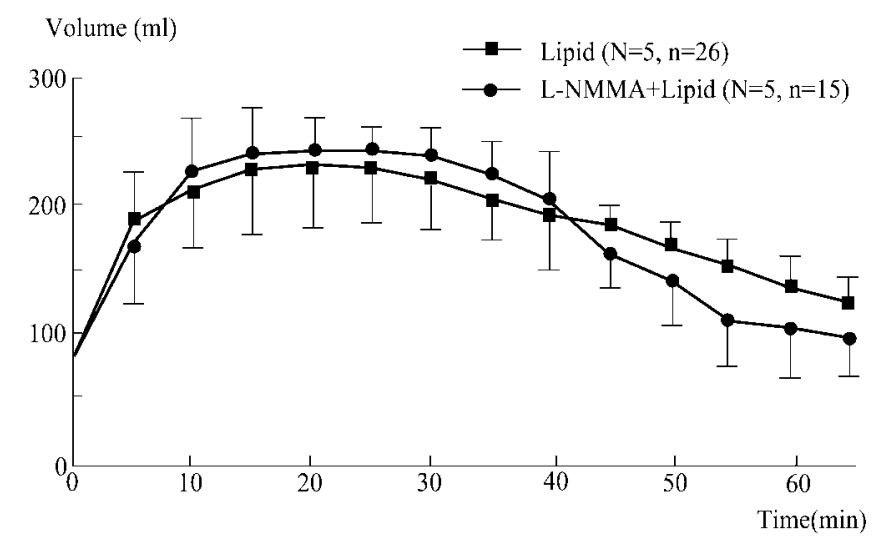

Fig. 7. Effect of L-NMMA on gastric relaxation induced by lipid ingestion. Post-prandial time course of the gastric relaxation response was similar in 2 groups throughout the experiment. The curve is based on 5-min average values.

milder from of gastric relaxation. This may suggest that gastric relaxation consequent upon water ingestion is caused by stimulation of the pharyngeal and esophageal mechanical receptors, whereas that consequent upon lipid ingestion or lipid infusion is caused by the stimulation of gastric chemical receptors in addition to purely mechanical receptors. We have in fact been able to confirm by fluoroscopy that lipid remains in the dog stomach for at least 20 minutes and only then begins its gradual transfer to the duodenum (data not shown). 
Several investigators have studied gastric relaxation in conscious dogs whose small bowels have been stimulated by chemicals (Azpiroz et al., 1985; Azpiroz et al., 1986; Mulemans et al., 1995). To our knowledge no one has yet studied the mechanisms involved in the gastric relaxation that flows from deliberately stimulating the stomach with chemicals. One study revealed that the intraduodenal infusion of lipid through a duodenal cannula can produce gastric relaxation, and that this response can be significantly attenuated by nitro-L-arginine (L-NNA) and reversed by L-arginine (Mulemans et al., 1995). The authors concluded that gastric relaxation induced by intraduodenal infusion of lipid was mediated by a feedback mechanism involving a neural or humoral pathway, and that NO was an important mediator of this mechanism. In our experiments, L-NMMA did not affect the gastric relaxation that stemmed from the ingestion of lipid. The disparity between our results and those reported by others may relate to the different sites of action involved. Our findings clearly indicate that nitrergic input is not essential for the gastric relaxation that can be induced by chemical stimulation in conscious dogs.

Previous investigations have shown that fasting gastric tone is maintained by continuous excitatory cholinergic input via the vagus nerve (Azpiroz et al., 1987; Jahnberg et al., 1977). A recent study in lightly anesthetized cats showed that $\mathrm{N}^{\omega}$-nitro-L-arginine methyl ester hydrochloride (L-NAME, $50 \mathrm{mg} / \mathrm{kg}$ i.v.) led to an increase in fasting gastric tone, and that this effect could be reversed by L-arginine, suggesting that resting proximal gastric tone is dependent not only on excitatory cholinergic input but also on a continuous inhibitory nitrergic input (Coulie et al., 1999). In our study, L-NMMA did not affect the baseline volume of the stomach. The reasons for this disparity could involve differences in animal species, amount and agent of NO synthase inhibitors.

The proximal stomach relaxes when food passes through the pharynx and esophagus, a response known as receptive relaxation (Cannon et al., 1911). When estimated with a barostat, receptive relaxation is manifested as a prompt fall in intragastric pressure after lipid or water ingestion. It does not appear to be an artifact due to movements of the dog. We were in fact monitoring movements of the dogs used in our experiments throughout, and we were able to detect a prompt fall in intragastric pressure when dogs drank a lipid meal or water even when they remained immobile. When the dogs did move, we observed irregular and much larger increases in gastric pressure. The prompt fall associated with receptive relaxation did not occur in the absence of pharyngeal and esophageal stimulation. Recent studies suggest that receptive relaxation is mediated by NO via vagal NANC inhibitory nerves in isolated guinea-pig or rat stomach (Desai et al., 1991) and in the anesthetized dog (Meulemans et al., 1995). However, we found that receptive relaxation still occurred after the administration of L-NMMA. This disparity may be a result of differences in our experimental approaches, since we note that the earlier studies utilized electrical stimulation of the vagus nerve as a way of inducing receptive relaxation. Our results clearly indicate that NO does not mediate receptive relaxation in conscious dogs.

In summary, our results appear to indicate that nitrergic input is necessary for mechanically induced gastric adaptive relaxation, but is not necessary for either gastric receptive relaxation or chemically induced gastric adaptive relaxation in conscious dogs. 


\section{Acknowledgments}

We are grateful to Prof. T. Asahara, Department of Surgery, Division of Frontier Medical Science, Programs for Biomedical Research, Graduate School of Biomedical Sciences, Hiroshima University, and Prof. K. Fujii, Department of Physiology, Hiroshima Jogakuin University, for valuable discussions and for kindly reviewing the manuscript. We also appreciate the helpful suggestions, operational guidance and animal care provided by Drs. M. Okajima, M. Arita, R. Kobayashi, S. Ikeda, H. Haneji and members of the GI Motility Research Group of Hiroshima University. We are grateful for the members of the Research Facilities for Laboratory Animal Sciences, Hiroshima University Faculty of Medicine. This work was supported by Grant-in-Aid for Scientific Research from Japanease Ministry of Education, Culture, Sports, Science and technology. Several parts of these experiments were presented at the annual meeting of the American Gastroenterological Association, Orlando, May 1999 and San Diego, May 2000.

\section{References}

Abrahamsson, H. (1973). Studies on the inhibitory nervous control of gastric motility. Acta Physiol. Scand. Suppl 390: 1-38.

Azpiroz, F. and Malagelada, J.R. (1985). Intestinal control of gastric tone. Am. J. Physiol. 249: G501G509.

Azpiroz, F. and Malagelada, J.R. (1985). Physiological variations in canine gastric tone measured by an electronic barostat. Am. J. Physiol. 248: G229-G237.

Azpiroz, F. and Malagelada, J.R. (1986). Vagally mediated gastric relaxation induced by intestinal nutrients in the dog. Am. J. Physiol. 251: G727-G735.

Azpiroz, F. and Malagelada, J.R. (1987). Importance of vagal input in maintaining gastric tone in the dog. J. Physiol. (Lond.) 384: 511-524.

Cannon, W.B. (1898). The movements of the stomach studied by means of the roentgen rays. Am. J. Physiol. 1: 359-382.

Cannon, W.B. and Lieb, C.W. (1911). The receptive relaxation of the stomach. Am. J. Physiol. 29: 267273.

Coffin, B., Azpiroz, F., Guarner, F. and Malagelada, J.R. (1994). Selective gastric hypersensitivity and reflex hyporeactivity in functional dyspepsia. Gastroenterol. 107: 1345-1351.

Coulie, B., Tack, J., Sifrim, D., Andrioli, A. and Janssens, J. (1999). Role of nitric oxide in fasting gastric fundus tone and in 5-HT1 receptor-mediated relaxation of gastric fundus. Am. J. Physiol. 276: G373-G377.

Desai, K.M., Zembowicz, A., Sessa, W.C. and Vane, J.R. (1991). Nitroxergic nerves mediate vagally induced relaxation in the isolated stomach of the guinea pig. Proc. Natl. Acad. Sci. U.S.A. 88: 11490-11494.

Desai, K.M., Sessa, W.C. and Vane, J.R. (1991). Involvement of nitric oxide in the reflex relaxation of the stomach to accommodate food or fluid. Nature 351: 477-479.

Hausken, T. and Berstad, A. (1994). Effect of glyceryl trinitrate on antral motility and symptoms in patients with functional dyspepsia. Scand. J. Gastroenterol. 29: 23-28.

Jahnberg, T., Abrahamsson, H., Jansson, G. and Martinson, J. (1977). Gastric relaxatory response to feeding before and after vagotomy. Scand. J. Gastroenterol. 12: 225-228.

Kim, D.Y., Delgado-Aros, S., Camilleri, M., Samsom, M., Murray, J.A., O’Connor, M.K., Brinkmann, B.H., Stephens, D.A., Lighvani, S.S. and Burton, D.D. (2001). Noninvasive measurement of 
gastric accommodation in patients with idiopathic nonulcer dyspepsia. Am. J. Gastroenterol. 96: 3099-3105.

Martinson, J. (1965). Vagal relaxation of the stomach. Experimental re-investigation of the concept of the transmission mechanism. Acta Physiol. Scand. 64: 453-462.

McLaughlin, J.T., Troncon, L.E., Barlow, J., Heggie, L.J. and Thompson, D.G. (1998). Evidence for a lipid specific effect in nutrient induced human proximal gastric relaxation. Gut 43: 248-251.

Meulemans, A.L., Eelen, J.G. and Schuurkes, J.A. (1995). NO mediates gastric relaxation after brief vagal stimulation in anesthetized dogs. Am. J. Physiol. 269: G255-G261.

Meulemans, A. and Schuurkes, J. (1995). Intralipid-induced gastric relaxation is mediated via NO. Neurogastroenterol. Motil. 7: 151-155.

Paterson, C.A., Anvari, M., Tougas, G. and Huizinga, J.D. (2000). Nitrergic and cholinergic vagal pathways involved in the regulation of canine proximal gastric tone: an in vivo study. Neurogastroenterol. Motil. 12: 301-306.

Ropert, A., des Varannes, S.B., Bizais, Y., Roze, C. and Galmiche, J.P. (1993). Simultaneous assessment of liquid emptying and proximal gastric tone in humans. Gastroenterol. 105: 667-674.

Troncon, L.E., Bennett, R.J., Ahluwalia, N.K. and Thompson, D.G. (1994). Abnormal intragastric distribution of food during gastric emptying in functional dyspepsia patients. Gut 35: 327-332.

Undeland, K.A., Hausken, T., Gilja, O.H., Ropert, R., Galmiche, J.P. and Berstad, A. (1995). Gastric relaxation in response to a soup meal in healthy subjects. A study using a barostat in the proximal stomach. Scand. J. Gastroenterol. 30: 1069-1076.

Uno, H., Arakawa, T., Fukuda, T., Higuchi, K., Hayakawa, T., Kase, Y., Takeda, S. and Kobayashi, K. (1995). Evaluation of gastric adaptive relaxation in isolated stomach from the guinea-pig. Jpn. J. Gastroenterol. 92: 1832-1838.

Villanova, N., Azpiroz, F. and Malagelada, J.R. (1997). Perception and gut reflexes induced by stimulation of gastrointestinal thermoreceptors in humans. J. Physiol. (Lond.) 502: 215-222.

Whitehead, W.E. and Delvaux, M. (1997). Standardization of barostat procedures for testing smooth muscle tone and sensory thresholds in the gastrointestinal tract. The Working Team of GlaxoWellcome Research, UK. Dig. Dis. Sci. 42: 223-241.

(Received February 2, 2004; Accepted March 19, 2004) 